\title{
Biomarkers of Sleep Apnea
}

\author{
Sydney B. Montesi, MD; Ednan K. Bajwa, MD, MPH; and Atul Malhotra, MD
}

\begin{abstract}
Obstructive sleep apnea (OSA) is a condition of repetitive upper airway collapse, which occurs during sleep. Recent literature has emphasized the role of OSA in contributing to glucose intolerance, dyslipidemia, and hypertension. OSA is associated with the development of cardiovascular disease, although definitive data are sparse with regard to the prevention of cardiovascular disease and CPAP therapy. CPAP provides effective treatment for OSA, but patient adherence remains challenging. Aside from daytime symptom improvement, it is difficult to monitor the adequacy of treatment response. Thus, the search for a biomarker becomes critical. The discovery of an ideal biomarker for OSA has the potential to provide information related to diagnosis, severity, prognosis, and response to treatment. In addition, because large-scale randomized controlled trials are both ethically and logistically challenging in assessing hard cardiovascular outcomes, certain biomarkers may be reasonable surrogate outcome measures. This article reviews the literature related to potential biomarkers of OSA with the recognition that an ideal biomarker does not exist at this time.

CHEST 2012; 142(1):239-245
\end{abstract}

Abbreviations: $\mathrm{AHI}=$ apnea-hypopnea index; $\mathrm{CRP}=\mathrm{C}$-reactive protein; $\mathrm{DM}=$ diabetes mellitus; HbAlc $=$ hemoglobin Alc; OSA $=$ obstructive sleep apnea; TNF- $\alpha=$ tumor necrosis factor- $\alpha$

O bstructive sleep apnea (OSA) is a condition characterized by repetitive collapse of the upper airway during sleep. Upper airway collapse leads to a reduction or cessation of airflow, impairing sleep continuity, autonomic function, and gas exchange. OSA is a common disorder affecting at least $2 \%$ to $4 \%$ of middle-aged women and men, respectively, according to the Wisconsin Sleep Cohort Study, ${ }^{1}$ but these OSA prevalence figures are likely underestimates of the current disease burden, which are based on increasing risk factors (eg, obesity, aging) plus improve-

Manuscript received September 9, 2011; revision accepted January 17, 2012.

Affiliations: From the Department of Pulmonary and Critical Care Medicine (Drs Montesi and Bajwa), Massachusetts General Hospital, and Department of Sleep Medicine (Dr Malhotra), Brigham and Women's Hospital, Boston, MA.

Funding/Support: Dr Bajwa is funded by National Institutes of Health [Grant K23 HL087934]. Dr Malhotra is funded by National Institutes of Health [Grants R01 AG035117, P01 HL095491, K24 HL093218, R01 HL090897, and R01 HL085188] and American Heart Association [Grant 0840159N].

Correspondence to: Sydney B. Montesi, MD, Department of Pulmonary and Critical Care Medicine, Massachusetts General Hospital, Bulfinch 148, 55 Fruit St, Boston, MA 02114; e-mail: sbmontesi@partners.org

(C) 2012 American College of Chest Physicians. Reproduction of this article is prohibited without written permission from the American College of Chest Physicians. See online for more details. DOI: $10.1378 /$ chest.11-2322 ments in diagnostic technology that have since occurred. Symptoms of excessive snoring, episodes of apnea, and daytime hypersomnolence suggest underlying sleep-disordered breathing; however, the diagnosis of OSA generally is made based on overnight polysomnography. Because polysomnography testing is both time and labor intensive and awareness of sleep disorders is relatively poor, OSA likely remains underdiagnosed. ${ }^{2}$

There is growing evidence to support the importance of effectively diagnosing and treating OSA as more is learned about its long-term effects. Hypoxemia caused by airway collapse, leading to nocturnal desaturation followed by reoxygenation, has been associated with surges in sympathetic activation, reactive oxygen species formation, increased inflammatory factors, and endothelial dysfunction. ${ }^{2}$ Although the complete impact of these effects is not fully understood, a growing body of literature shows associations between OSA and glucose intolerance, dyslipidemia, and hypertension. Data support a link between OSA and cardiovascular disease, ${ }^{3,4}$ and further study is needed to assess whether this association is causal or a result of comorbid medical conditions. The growing knowledge of OSA as a contributor to hypertension (both systemic and within the pulmonary circulation) and glucose intolerance provides additional 
motivation for appropriate diagnosis and treatment of OSA.

OSA is effectively diagnosed through the use of polysomnography and treated with CPAP. However, OSA may be underdiagnosed because of nonrecognition of signs and symptoms by health-care providers as well as hesitancy of patients to follow through on overnight polysomnography and eventual therapy. Even when OSA is diagnosed, issues such as poor patient adherence can prevent adequate treatment with CPAP overnight. In addition, no perfect definition of OSA exists because existing metrics correlate loosely with outcome, and different desaturation thresholds are likely required for various different outcome measures (eg, $\mathrm{BP}$ vs insulin resistance). ${ }^{5}$ Thus, the quest for OSA biomarkers is critical.

Recently, Shih and Malhotra ${ }^{6}$ described requisite characteristics for an ideal biomarker for sleep apnea, citing that the optimal biomarkers would have use as a diagnostic measure, a means for assessing disease burden and severity, and a method for measuring response to treatment. For diagnostic utility, an ideal biomarker would need to be both sensitive and specific, which in theory, could obviate the need for polysomnography, at least in some patients (Table 1). For the biomarker to be useful in tracking response to therapy, it should be involved in the pathogenesis of complications and should respond to treatment. If the biomarker were on a causal pathway known to be important in disease complications, changes in biomarker levels in response to therapy could be viewed as reliably predictive of reduced complications, allowing the ideal biomarker to be used as a surrogate outcome measure in clinical trials. Because long-term randomized controlled trials are both logistically and ethically challenging, a biomarker with such characteristics would be a welcome addition.

This article reviews the present data on potential biomarkers that may be used in the diagnosis and treatment monitoring of OSA, recognizing that no ideal biomarker currently exists. However, we refer the interested reader to more comprehensive reviews regarding topics not discussed here, including newer cardiac biomarkers, ${ }^{7}$ pediatric sleep apnea ${ }^{8}$ and invasive metabolic testing. ${ }^{9}$

\section{MARKERS OF INFLAMMATION}

OSA has been associated with elevations in biochemical markers of inflammation, a state that is conjectured to contribute to the increased risk of cardiovascular disease. Although the exact mechanism is not known, both sleep deprivation and hypoxemia are believed to be important causative factors. ${ }^{10-12}$ In addition, adipose tissue can produce proinflammatory markers (adipokines), such as tumor necrosis- $\alpha$ $(\mathrm{TNF}-\alpha)$ and IL-6, and obesity is a common comorbid condition with OSA. ${ }^{10}$ Inflammatory marker elevation has been shown in healthy individuals in the setting of sleep deprivation. ${ }^{11}$ A significant rise of IL-6 levels in both men and women and TNF- $\alpha$ levels in men alone was observed after 7 days of a $25 \%$ reduction in the amount of overnight sleep in otherwise healthy, young, and nonobese (average BMI, $23.8 \mathrm{~kg} / \mathrm{m}^{2}$ ) subjects. ${ }^{11}$ Elevations in IL-6, C-reactive protein (CRP), and $\mathrm{TNF}-\alpha$ were seen with a decrease in sleep duration by $1 \mathrm{~h}$ as assessed by polysomnography; however, this observed increase was attenuated in all but $\mathrm{TNF}-\alpha$ results when adjusted for confounders such as waist circumference and BMI. ${ }^{12}$ Steiropoulos et $\mathrm{al}^{13} \mathrm{com}-$ pared levels of inflammatory markers CRP, TNF- $\alpha$, IL-6, and fibrinogen in obese patients with and without OSA. Subjects included men and women who were matched by both waist circumference and BMI. Levels of TNF- $\alpha$ alone were significantly elevated in obese subjects with OSA compared with those without OSA. Of note, significant increases in CRP levels were seen with both minimum and average values of oxygen saturation as measured by pulse oximetry, lending support to the idea of hypoxemia as the causative trigger for inflammatory markers.

The relationship between CRP and OSA is difficult to define conclusively, given contradictory reports in the literature. ${ }^{12-16}$ In a study of 22 patients with untreated OSA, elevations in CRP were associated with disease severity compared with control subjects matched for

Table 1-Ideal Biomarker Characteristics

\begin{tabular}{|c|c|}
\hline Ideal Biomarker & Comment \\
\hline Sensitive for disease & Screening test, diagnostic utility \\
\hline Specific for disease & Few false positives avoid unnecessary PSG \\
\hline Dose responsive, correlates with disease severity & Could quantify disease burden, prioritize therapy \\
\hline Treatment responsive & Use as a metric for adequacy of therapy or adherence to CPAP \\
\hline Involvement in important causal pathway & Reliable surrogate outcome measure, predicting disease complications \\
\hline Easily measured & Would not require major expertise to assess \\
\hline Inexpensive & Allow high throughput in clinic or research \\
\hline Panel of metrics & Assess multiple pathways, eg, inflammation, oxidative stress, autonomic \\
\hline
\end{tabular}

PSG $=$ polysomnography. 
factors including BMI, sex, and age. ${ }^{14}$ Similarly, in a large prospective cohort study of 3,888 subjects in Japan, high-sensitivity CRP values increased in parallel with the degree of overnight hypoxemia, even with adjusting for BMI and other cardiovascular risk factors. ${ }^{15}$ However, in other studies, elevations in CRP in the setting of sleep deprivation or OSA either were not found or were not significant after adjusting for measures of obesity. ${ }^{12,13}$

If hypoxemia is believed to be the provoking factor for the inflammatory cascade, then CPAP, by eliminating episodes of recurrent desaturations, should theoretically limit the release of inflammatory factors. ${ }^{17}$ In another study by Steiropoulos et al, ${ }^{18}$ high-sensitivity CRP levels along with levels of total cholesterol, highdensity lipoprotein, low-density lipoprotein, apolipoprotein AI, apolipoprotein B, and homocysteine were obtained at the initiation of CPAP therapy for newly diagnosed OSA. At 6 months, a statistically significant decrease was seen in high-sensitivity CRP, total cholesterol, and homocysteine levels for subjects who adhered to at least $4 \mathrm{~h}$ of CPAP use per night. Along these lines, a randomized controlled crossover study of 30 subjects with OSA found a decrease in TNF- $\alpha$ receptor 1 values after 3 months of overnight CPAP use. ${ }^{19}$ In contrast, a larger randomized controlled trial of 100 men receiving therapeutic vs subtherapeutic CPAP (defined as adequate vs inadequate pressure for pharyngeal opening) did not show a change in highsensitivity CRP or IL-6 levels after 4 weeks of therapy. ${ }^{20}$ Further studies are needed to determine the effect CPAP has on reducing elevations in inflammatory markers and the optimal duration of CPAP therapy needed to achieve such a result. In theory, certain subsets of patients (eg, defined by race, genetics, disease severity) may be particularly susceptible to a proinflammatory state, emphasizing the need for larger data sets.

Despite these data, use of inflammatory factors as diagnostic markers for sleep-disordered breathing remains challenging. Although markers like CRP can assess for systemic inflammation, they can be elevated in a myriad of inflammatory conditions. Because OSA often is a comorbid illness, it would be challenging to separate out elevations in inflammatory biomarkers attributable to OSA alone, short of using randomized controlled trials of CPAP therapy. Further data may be able to elucidate an avenue in which inflammatory markers may be useful either diagnostically or as a means to stage severity. In the meantime, additional inquiry into the role of inflammation could provide further clues about the pathogenesis of cardiovascular disease in OSA.

\section{HyPERTENSION AS AN OSA BIOMARKER}

Although the relationship between OSA and cardiovascular disease needs further study, there is consid- erable information supporting OSA as an independent risk factor for cardiovascular disease. ${ }^{4}$ The exact role of OSA in pathogenesis is difficult to discern because a large proportion of patients with OSA also have hypertension, obesity, and diabetes mellitus (DM), which independently confer cardiac risk.

Of recent interest has been the causal link between sleep-disordered breathing and hypertension, primarily systemic hypertension. ${ }^{10}$ The proposed underlying mechanism is believed to be due to sympathoexcitation, which occurs during episodes of apnea and oxygen desaturation. ${ }^{10}$ Brooks et al ${ }^{21}$ induced OSA in four canine animals and showed that OSA caused elevations not only in nightly BP but also in daytime readings, with changes seen as early as 4 weeks from the induction of OSA. Elevations in BP resolved in the absence of OSA, with daytime BP elevations taking up to 3 weeks to return to pre-OSA values. The relationship between sleep apnea and hypertension may be especially significant in light of the role of OSA in cardiovascular disease. Studies have been conflicting about whether OSA is an independent risk factor for hypertension. The Sleep Heart Health Study ${ }^{22}$ prospectively compared BP values with apnea-hypopnea index (AHI) values. A linear relationship between $\mathrm{BP}$ and $\mathrm{AHI}$ was observed at 5 years; however, the effect was abrogated when the ORs of the results were adjusted for obesity, as determined by BMI. Conversely, Peppard et al ${ }^{23}$ found a significant relationship between hypertension and AHI over a 4-year period, with increasing ORs paralleling the rise in AHI. Significance was achieved even after adjusting for major covariates, including obesity through BMI and waist circumference.

Use of CPAP has been shown to have an effect, albeit modest, on patients with OSA from the standpoint of BP. Barbé et al $^{24}$ assessed changes in BP during long-term treatment with CPAP in patients with both OSA and hypertension. Decreases in systolic and diastolic BP by 1.89 and $2.19 \mathrm{~mm} \mathrm{Hg}$, respectively, were noted after 12 months of overnight CPAP therapy, although statistical significance was reached only for lowering of diastolic BP. The effect was independent of BMI and most pronounced when CPAP was used for $>5.6 \mathrm{~h}$ each night. Further, $\mathrm{BP}$ reduction was demonstrated in patients who had severe OSA but no symptoms of excessive somnolence during the day. In addition, Durán-Cantolla et al ${ }^{25}$ used 24-h ambulatory BP monitoring devices to assess $\mathrm{BP}$ values among patients with moderate to severe OSA and hypertension who were not on antihypertensive therapy. A significant decrease in mean systolic $\mathrm{BP}$ by $2.1 \mathrm{~mm} \mathrm{Hg}$ and mean diastolic BP by $1.3 \mathrm{~mm} \mathrm{Hg}$ was noted after 12 weeks in those patients randomized to receive nocturnal CPAP.

Although CPAP does have BP-lowering effects, more studies are needed to determine the optimal 
duration of overnight CPAP use associated with maximum BP-lowering benefit. Antihypertensive therapy still remains the first-line therapy for hypertension in those with sleep-disordered breathing. In a recent randomized controlled crossover trial, CPAP was compared with valsartan in patients with OSA and untreated hypertension. ${ }^{26}$ CPAP alone produced a mean $\mathrm{BP}$ reduction of $2.1 \mathrm{~mm} \mathrm{Hg}$ compared with valsartan, which led to a mean reduction of $7.4 \mathrm{~mm} \mathrm{Hg}$. The increased efficacy of valsartan may be due to the blocking of the renin-angiotensin cascade triggered by hypoxemia. ${ }^{26}$ Regardless, daytime BP alone is unlikely to motivate patients to use CPAP.

CPAP appears to have a favorable benefit on lowering $\mathrm{BP}$, and the use of CPAP in addition to antihypertensive therapy has the potential to result in additional BP-lowering effects, particularly nocturnal surges in BP associated with apnea. ${ }^{26}$ Although the observed improvement in BP with OSA therapy suggests only modest effects, we would emphasize several points. First, the BP improvements seen in patients with drug-refractory hypertension may be more substantial, at least according to some studies. ${ }^{27}$ Second, some authors have suggested that vasoregulatory mechanisms will minimize changes in $\mathrm{BP}$ achieved through changes in vasoconstriction or vasodilation, emphasizing the importance of central control of BP in determining daytime resting set point values. ${ }^{28}$ Thus, vasoregulatory mechanisms may ensure a modest change in $\mathrm{BP}$ with reduced vasoconstriction. Third, nocturnal surges in BP are rarely captured with noninvasive testing but are easily observed during repetitive apnea when an indwelling arterial line is in place. As such, daytime BP is simply a surrogate outcome measure for cardiovascular risk, whereas nocturnal surges in BP may be a more important stimulus for plaque rupture and hard cardiovascular outcomes. ${ }^{29}$ Thus, minor changes in daytime BP with CPAP therapy may underestimate the therapeutic benefit. Other mechanisms independent of BP (eg, oxidative stress as described later in this article) may cause more critical complications in some patients with OSA. Fourth, given the phenotypic variability in expression of disease, there are likely to be some patients in whom CPAP has a major benefit from the standpoint of BP improvement. Ongoing efforts may help to define genetic or demographic factors that define the "big responders." Finally, although subset analyses show better BP improvements in patients who are most adherent with CPAP, such analyses are complicated by the healthy user effect such that highly compliant CPAP users may be the most motivated, educated patients whose outcome is good for reasons independent of airway pressure. ${ }^{30}$ Such data also emphasize the importance of developing new therapies for OSA to help reduce risk among those patients incompletely addressed with CPAP.

\section{CARotid InTIMAL-Medial Thickness}

OSA has been associated with the risk of stroke in both cross-sectional ${ }^{31}$ and longitudinal studies. ${ }^{32-35}$ The mechanism underlying stroke has not been fully elucidated but likely is multifactorial, including cardioembolic risk, vibration-induced carotid injury, and promotion of atherothrombosis in the carotid arteries. The cardioembolic risk is likely mediated by impairments in left ventricular function ${ }^{36}$ as well as in atrial fibrillation. ${ }^{37}$ Snoring has been associated with endothelial injury, akin to vibration-induced endothelial dysfunction in jackhammer users. ${ }^{38-40}$ Some have speculated that vibration may mechanically injure existing plaque, leading to distal embolization. OSA has been linked to atherothrombosis based on studies of pulse-wave velocity as well as intimal-medial thickness showing a dose-response relationship of vascular compromise and severity of OSA ${ }^{41}$ Similarly, studies of platelet function have shown that degree of hypoxemia is associated with glycoprotein Ib expression on the surface of platelets, with greater hypoxemia linked to evidence of platelet activation. ${ }^{42}$ Thus, a strong biologic basis exists for a causal link between OSA and cerebrovascular disease, although randomized trials are needed for definitive interventional data.

\section{Metabolic Markers}

Of the metabolic changes caused by OSA, among the most discussed is glucose metabolism. Recent literature supports an important effect of sleepdisordered breathing on glucose regulation. A strong association exists between DM and OSA, with a large proportion of obese patients having both conditions. The recent Sleep AHEAD (Action for Health in Diabetes) study screened obese patients with known type $2 \mathrm{DM}$ for OSA by overnight polysomnography. ${ }^{43}$ Of the $>300$ patients who underwent polysomnography, $86.6 \%$ met criteria for OSA. With obesity being a risk factor for both DM and OSA, the question exists whether BMI is a confounder to explain the relationship between OSA and impaired glycemia. Despite the large proportion of patients with OSA with coexisting obesity, the effect of OSA on glucose intolerance has been shown to be independent of BMI, at least in some studies. ${ }^{44,45}$

In patients with known DM, the presence of mild, moderate, and severe OSA raised hemoglobin Alc (HbAlc) levels by an average of $1.49 \%, 1.93 \%$, and $3.69 \%$, respectively, with the elevation in HbAlc noted independently of BMI. ${ }^{44} \mathrm{HbAlc}$ abnormalities are seen not just in patients with underlying glucose 
intolerance or diabetes. Papanas et al ${ }^{45}$ showed that in patients with OSA but without DM, there was a significant linear correlation between arousal index and HbAlc and fasting glucose levels, and again, this effect was independent of obesity. The degree of glucose impairment is associated with OSA severity because increased glucose levels and pancreatic $\beta$-cell activity have been associated with severity of OSA in patients with normal glucose metabolism. ${ }^{46}$ Based on these data, level of HbAlc can be viewed as a potential biomarker for OSA. Although the current clinical use of HbAlc level is limited to the diagnosis and monitoring of DM, with time it may be shown to have utility for managing certain patients with OSA. On the other hand, therapeutic studies of OSA in DM have shown variable effects on glycemic control, emphasizing the need to define in which patients $\mathrm{HbAlC}$ may be a useful biomarker. Until then, given the overlapping spectrum of OSA and DM and the effects of OSA on HbAlc levels in patients with and without underlying glucose impairment, HbAlc testing could be considered to assess for DM in those with OSA. ${ }^{47}$ Patients with both OSA and DM may be at high risk of vascular complications ${ }^{48}$ suggesting that these patients may be particularly likely to benefit from aggressive intervention.

\section{MARKers of OXIDATIVE STRESS}

Oxidative stress has been studied extensively for its relationship to atherosclerosis and cardiovascular disease and has emerged as a key component in the pathogenesis of OSA. Episodes of hypoxemia secondary to repetitive airway obstruction and intermittent apnea and subsequent reoxygenation are conjectured to trigger the formation of free radical species, thus igniting a cascade of oxidative stress marker production. ${ }^{49}$ Emerging evidence suggests that these pathways of increased oxidative stress are at least partially responsible for the deleterious metabolic and cardiovascular side effects of OSA. ${ }^{50,51}$ The development of excess free radical formation and markers of increased oxidative stress are the unifying paradigm, as suggested by Lavie, ${ }^{51}$ by which OSA results in cardiovascular disease and metabolic impairment.

Data have revealed altered levels of markers of oxidative stress in patients with OSA. Clinical interest has focused on 8-isoprostane, IL-6, and nitric oxide. Studies of exhaled breath condensate have found elevations in 8-isoprostane and IL-6 in patients with OSA and other respiratory disorders. ${ }^{52-54}$ Carpagnano et al ${ }^{53}$ compared levels of 8-isoprostane and IL-6 through immunoassay of exhaled breath condensate among subjects with OSA, subjects who were obese, and healthy control subjects. Although the total study population was only 43 patients, levels of IL- 6 and 8-isoprostane, though elevated in those with obesity, were highest in the group with OSA. A follow-up study by the same group of investigators assessed the effect of overnight CPAP on serum and exhaled breath condensate levels of 8-isoprostane in subjects with OSA. ${ }^{54}$ Elevations in 8-isoprostane were seen in both serum and exhaled breath condensate at 8:00 $\mathrm{AM}$ in a study group of 18 subjects with OSA (compared with 12 weight-matched control subjects without OSA), and these levels were significantly reduced after overnight use of CPAP. The degree of exhaled breath condensate 8-isoprostane morning values had a direct correlation with neck circumference and AHI.

Alonso-Fernández et al ${ }^{55}$ performed a randomized cross-over study to assess the change in serum 8-isoprostane and nitrite levels in OSA after 12 weeks of overnight CPAP therapy. They randomized male subjects with OSA and matched control subjects to receive therapeutic vs sham CPAP. All subjects with underlying cardiovascular or pulmonary disease aside from OSA were excluded. Compared with the control group, initial values were higher for 8-isoprostane and lower for nitrite in the study population; however, 12 weeks of CPAP therapy was observed to lower 8-isoprostane levels and raise nitrite levels to that of the control subjects. Although further studies are needed, these data point to the potential use of 8 -isoprostane as a biomarker for OSA and add credibility to the notion that oxidative stress may contribute to OSA complications.

\section{Other Potential Markers of OSA}

Cysteine has emerged as a possible biomarker of OSA. Both cysteine and homocysteine have been studied in relation to cardiovascular disease ${ }^{56}$ and recently in OSA. ${ }^{57,58}$ Cintra et al ${ }^{58}$ examined levels of cysteine and homocysteine in 75 sex- and agematched subjects with and without OSA. Levels of homocysteine were similar in both groups, but cysteine levels were higher in the OSA group. Although the two study groups were not matched for obesity, elevation in cysteine in the OSA group was still present, with adjustment for BMI. For 15 subjects, plasma cysteine and homocysteine levels were obtained at initiation of CPAP therapy and after 1 and 6 months of treatment. A significant decrease in cysteine alone was noted with CPAP administration over the 6-month interval. Additional studies are needed on a larger scale to delineate the relation of cysteine levels and OSA and the influence of treatment.

\section{CONCLUSIONS}

OSA is a common disorder of which the effects far exceed the transient ventilatory impairment caused 
by upper airway collapse. Emerging data continue to demonstrate that sleep-disordered breathing has both cardiovascular and metabolic consequences, ${ }^{59}$ and improvements in diagnosis and treatment come to the forefront of efforts to help negate the harmful consequences of this disease. Although several candidate biomarkers have emerged that show promise for improving methods of diagnosis and treatment, none has yet warranted consideration as an ideal biomarker. ${ }^{5}$ Additional work remains to be done on this important topic because various biomarkers (or combinations of biomarkers) may be useful for capturing different manifestations of disease (eg, markers of oxidative stress vs hypoxic burden vs sympathoexcitation). Such research would then be helpful in the design of interventional studies using antiinflammatory agents, antioxidants, or both ${ }^{60}$ to help to understand how apnea complications can be modulated pharmacologically. We would predict that combinations of biomarkers will likely be required to predict the myriad of recognized OSA complications. In addition, ongoing efforts using proteomics ${ }^{61}$ and other unbiased approaches (before and after treatment or before, during, and after sleep) may help to define potential biomarkers of the future for OSA.

\section{ACKNOWLEDGMENTS}

Financial/nonfinancial disclosures: The authors have reported to CHEST the following conflicts of interest: Dr Malhotra received research and consulting income from Philips Respironics; Apnex Medical, Inc; Apnicure, Inc; Galleon Pharmaceuticals, Inc; Pfizer, Inc; Merck \& Co, Inc; Cephalon, Inc; Sunovion Pharmaceuticals, Inc; Ethicon, Inc; Medtronic, Inc; Sleep Group Solutions, Inc; and Sleep HealthCenters. Drs Montesi and Bajwa have reported that no potential conflicts of interest exist with any companies/organizations whose products or services may be discussed in this article.

Role of sponsors: The sponsors had no role in the design of the study, the collection and analysis of the data, or in the preparation of the manuscript.

\section{REFERENCES}

1. Young T, Palta M, Dempsey J, Skatrud J, Weber S, Badr S. The occurrence of sleep-disordered breathing among middleaged adults. N Engl J Med. 1993;328(17):1230-1235.

2. Kapur VK. Obstructive sleep apnea: diagnosis, epidemiology, and economics. Respir Care. 2010;55(9):1155-1167.

3. Malhotra A, Loscalzo J. Sleep and cardiovascular disease: an overview. Prog Cardiovasc Dis. 2009;51(4):279-284

4. Eckert DJ, Malhotra A. Pathophysiology of adult obstructive sleep apnea. Proc Am Thorac Soc. 2008;5(2):144-153.

5. Punjabi NM, Sorkin JD, Katzel LI, Goldberg AP, Schwartz AR, Smith PL. Sleep-disordered breathing and insulin resistance in middle-aged and overweight men. Am J Respir Crit Care Med. 2002;165(5):677-682

6. Shih JL, Malhotra A. Could vitamins be helpful to patients with sleep apnea? Chest. 2011;139(2):237-238.

7. Armstrong EJ, Morrow DA, Sabatine MS. Inflammatory biomarkers in acute coronary syndromes: part I: introduction and cytokines. Circulation. 2006;113(6):e72-e75.
8. Gozal D, Kheirandish-Gozal L. Cardiovascular morbidity in obstructive sleep apnea: oxidative stress, inflammation, and much more. Am J Respir Crit Care Med. 2008;177(4):369-375.

9. Punjabi NM, Beamer BA. Alterations in glucose disposal in sleep-disordered breathing. Am J Respir Crit Care Med. 2009;179(3):235-240.

10. Mullington JM, Haack M, Toth M, Serrador JM, MeierEwert HK. Cardiovascular, inflammatory, and metabolic consequences of sleep deprivation. Prog Cardiovasc Dis. 2009; 51(4):294-302

11. Vgontzas AN, Zoumakis E, Bixler EO, et al. Adverse effects of modest sleep restriction on sleepiness, performance, and inflammatory cytokines. J Clin Endocrinol Metab. 2004; 89(5):2119-2126.

12. Patel SR, Zhu X, Storfer-Isser A, et al. Sleep duration and biomarkers of inflammation. Sleep. 2009;32(2):200-204.

13. Steiropoulos P, Papanas N, Nena E, et al. Inflammatory markers in middle-aged obese subjects: does obstructive sleep apnea play a role? Mediators Inflamm. 2010;2010:675320.

14. Shamsuzzaman ASM, Winnicki M, Lanfranchi P, et al. Elevated C-reactive protein in patients with obstructive sleep apnea. Circulation. 2002;105(21):2462-2464.

15. Muraki I, Tanigawa T, Yamagishi K, et al; CIRCS Investigators. Nocturnal intermittent hypoxia and $\mathrm{C}$ reactive protein among middle-aged community residents: a cross-sectional survey. Thorax. 2010;65(6):523-527.

16. Lui MM, Lam JC, Mak HK, et al. C-reactive protein is associated with obstructive sleep apnea independent of visceral obesity. Chest. 2009;135(4):950-956.

17. Wu KM, Lin CC, Chiu CH, Liaw SF. Effect of treatment by nasal continuous positive airway pressure on serum high mobility group box-1 protein in obstructive sleep apnea. Chest. 2010;137(2):303-309.

18. Steiropoulos P, Tsara V, Nena E, et al. Effect of continuous positive airway pressure treatment on serum cardiovascular risk factors in patients with obstructive sleep apnea-hypopnea syndrome. Chest. 2007;132(3):843-851.

19. Arias MA, García-Río F, Alonso-Fernández A, et al. CPAP decreases plasma levels of soluble tumour necrosis factoralpha receptor 1 in obstructive sleep apnoea. Eur Respir J. 2008;32(4):1009-1015.

20. Kohler M, Ayers L, Pepperell JCT, et al. Effects of continuous positive airway pressure on systemic inflammation in patients with moderate to severe obstructive sleep apnoea: a randomised controlled trial. Thorax. 2009;64(1):67-73.

21. Brooks D, Horner RL, Kozar LF, Render-Teixeira CL, Phillipson EA. Obstructive sleep apnea as a cause of systemic hypertension. Evidence from a canine model. J Clin Invest. 1997;99(1):106-109.

22. O'Connor GT, Caffo B, Newman AB, et al. Prospective study of sleep-disordered breathing and hypertension: the Sleep Heart Health Study. Am J Respir Crit Care Med. 2009; 179(12):1159-1164

23. Peppard PE, Young T, Palta M, Skatrud J. Prospective study of the association between sleep-disordered breathing and hypertension. N Engl J Med. 2000;342(19):1378-1384.

24. Barbé F, Durán-Cantolla J, Capote F, et al; Spanish Sleep and Breathing Group. Long-term effect of continuous positive airway pressure in hypertensive patients with sleep apnea. Am J Respir Crit Care Med. 2010;181(7):718-726.

25. Durán-Cantolla J, Aizpuru F, Montserrat JM, et al; Spanish Sleep and Breathing Group. Continuous positive airway pressure as treatment for systemic hypertension in people with obstructive sleep apnoea: randomised controlled trial. BMJ. 2010;341:c5991.

26. Pépin JL, Tamisier R, Barone-Rochette G, Launois SH, Lévy P, Baguet JP. Comparison of continuous positive airway 
pressure and valsartan in hypertensive patients with sleep apnea. Am J Respir Crit Care Med. 2010;182(7):954-960.

27. Logan AG, Tkacova R, Perlikowski SM, et al. Refractory hypertension and sleep apnoea: effect of CPAP on blood pressure and baroreflex. Eur Respir J. 2003;21(2):241-247.

28. Guyenet PG. The sympathetic control of blood pressure. Nat Rev Neurosci. 2006;7(5):335-346.

29. Stradling J, Davies RJ. Sleep apnea and hypertension-what a mess! Sleep. 1997;20(9):789-793.

30. Platt AB, Kuna ST, Field SH, et al. Adherence to sleep apnea therapy and use of lipid-lowering drugs: a study of the healthyuser effect. Chest. 2010;137(1):102-108.

31. Shahar E, Whitney CW, Redline S, et al. Sleep-disordered breathing and cardiovascular disease: cross-sectional results of the Sleep Heart Health Study. Am J Respir Crit Care Med. 2001;163(1):19-25.

32. Yaggi HK, Concato J, Kernan WN, Lichtman JH, Brass LM, Mohsenin V. Obstructive sleep apnea as a risk factor for stroke and death. N Engl J Med. 2005;353(19):2034-2041.

33. Arzt M, Young T, Finn L, Skatrud JB, Bradley TD. Association of sleep-disordered breathing and the occurrence of stroke. Am J Respir Crit Care Med. 2005;172(11):1447-1451.

34. Redline S, Yenokyan G, Gottlieb DJ, et al. Obstructive sleep apnea-hypopnea and incident stroke: the sleep heart health study. Am J Respir Crit Care Med. 2010;182(2):269-277.

35. Djonlagic I, Malhotra A. Risk of stroke from sleep apnea in men and women. Expert Rev Neurother. 2010;10(8):1267-1271.

36. Gottlieb DJ, Yenokyan G, Newman AB, et al. Prospective study of obstructive sleep apnea and incident coronary heart disease and heart failure: the sleep heart health study. Circulation. 2010;122(4):352-360.

37. Gami AS, Hodge DO, Herges RM, et al. Obstructive sleep apnea, obesity, and the risk of incident atrial fibrillation. J Am Coll Cardiol. 2007;49(5):565-571.

38. Rice TB, Strollo PJ Jr. A nuisance or nemesis: the adverse effects of snoring. Sleep. 2011;34(6):693-694.

39. Rahangdale S, Campana L, Malhotra A. Not so good vibrations. Commentary on Lee et al. Heavy snoring as a cause of carotid artery atherosclerosis. SLEEP 2008;31(9):1207-1213. Sleep. 2008;31(9):1204-1205.

40. Cho JG, Witting PK, Verma M, et al. Tissue vibration induces carotid artery endothelial dysfunction: a mechanism linking snoring and carotid atherosclerosis? Sleep. 2011;34(6):751-757.

41. Drager LF, Bortolotto LA, Lorenzi MC, Figueiredo AC, Krieger EM, Lorenzi-Filho G. Early signs of atherosclerosis in obstructive sleep apnea. Am J Respir Crit Care Med. 2005; 172(5):613-618.

42. Rahangdale S, Yeh SY, Novack V, et al. The influence of intermittent hypoxemia on platelet activation in obese patients with obstructive sleep apnea. J Clin Sleep Med. 2011;7(2):172-178.

43. Foster GD, Sanders MH, Millman R, et al; Sleep AHEAD Research Group. Obstructive sleep apnea among obese patients with type 2 diabetes. Diabetes Care. 2009;32(6): 1017-1019.

44. Aronsohn RS, Whitmore H, Van Cauter E, Tasali E. Impact of untreated obstructive sleep apnea on glucose control in type 2 diabetes. Am J Respir Crit Care Med. 2010;181(5): 507-513.
45. Papanas N, Steiropoulos P, Nena E, et al. HbAlc is associated with severity of obstructive sleep apnea hypopnea syndrome in nondiabetic men. Vasc Health Risk Manag. 2009;5: 751-756.

46. Pallayova M, Steele KE, Magnuson TH, et al. Sleep apnea predicts distinct alterations in glucose homeostasis and biomarkers in obese adults with normal and impaired glucose metabolism. Cardiovasc Diabetol. 2010;9:83.

47. American Diabetes Association. Executive summary: standards of medical care in diabetes-2011. Diabetes Care. 2011; 34(suppl 1):S4-S10.

48. Yim-Yeh S, Rahangdale S, Nguyen ATD, et al. Vascular dysfunction in obstructive sleep apnea and type 2 diabetes mellitus. Obesity (Silver Spring). 2011;19(1):17-22.

49. Lavie L. Obstructive sleep apnoea syndrome-an oxidative stress disorder. Sleep Med Rev. 2003;7(1):35-51.

50. Suzuki YJ, Jain V, Park AM, Day RM. Oxidative stress and oxidant signaling in obstructive sleep apnea and associated cardiovascular diseases. Free Radic Biol Med. 2006;40(10): 1683-1692.

51. Lavie L. Oxidative stress - a unifying paradigm in obstructive sleep apnea and comorbidities. Prog Cardiovasc Dis. 2009;51(4):303-312.

52. Montuschi P, Kharitonov SA, Ciabattoni G, et al. Exhaled 8 -isoprostane as a new non-invasive biomarker of oxidative stress in cystic fibrosis. Thorax. 2000;55(3):205-209.

53. Carpagnano GE, Kharitonov SA, Resta O, Foschino-Barbaro MP, Gramiccioni E, Barnes PJ. Increased 8-isoprostane and interleukin-6 in breath condensate of obstructive sleep apnea patients. Chest. 2002;122(4):1162-1167.

54. Carpagnano GE, Kharitonov SA, Resta O, FoschinoBarbaro MP, Gramiccioni E, Barnes PJ. 8-Isoprostane, a marker of oxidative stress, is increased in exhaled breath condensate of patients with obstructive sleep apnea after night and is reduced by continuous positive airway pressure therapy. Chest. 2003; 124(4):1386-1392.

55. Alonso-Fernández A, García-Río F, Arias MA, et al. Effects of CPAP on oxidative stress and nitrate efficiency in sleep apnoea: a randomised trial. Thorax. 2009;64(7):581-586.

56. van den Brandhof WE, Haks K, Schouten EG, Verhoef P. The relation between plasma cysteine, plasma homocysteine and coronary atherosclerosis. Atherosclerosis. 2001;157(2):403-409.

57. Svatikova A, Wolk R, Magera MJ, Shamsuzzaman AS, Phillips BG, Somers VK. Plasma homocysteine in obstructive sleep apnoea. Eur Heart J. 2004;25(15):1325-1329.

58. Cintra F, Tufik S, D’Almeida V, et al. Cysteine: a potential biomarker for obstructive sleep apnea. Chest. 2011;139(2): 246-252.

59. Sharma SK, Agrawal S, Damodaran D, et al. CPAP for the metabolic syndrome in patients with obstructive sleep apnea. N Engl J Med. 2011;365(24):2277-2286.

60. Grebe M, Eisele HJ, Weissmann N, et al. Antioxidant vitamin $\mathrm{C}$ improves endothelial function in obstructive sleep apnea. Am J Respir Crit Care Med. 2006;173(8):897-901.

61. Gozal D, Jortani S, Snow AB, et al. Two-dimensional differential in-gel electrophoresis proteomic approaches reveal urine candidate biomarkers in pediatric obstructive sleep apnea. Am J Respir Crit Care Med. 2009;180(12):1253-1261. 